\title{
Use of video technology for teaching in intensive care
}

\author{
I Carroll ${ }^{1 *}$, N Thomas ${ }^{2}$, J Moore ${ }^{3}$ \\ From ESICM LIVES 2015 \\ Berlin, Germany. 3-7 October 2015
}

\section{Introduction}

Intensive care units in the UK are staffed by junior doctors with a range of backgrounds (anaesthesia, emergency and general medicine), often on rotations. Training is provided for practical procedures with an assessment of performance prior to independent practice.

There are national guidelines in place for the insertion and use of central venous catheters (CVCs) $[1,2]$ however complications persist. The national rate for CVC infection rate is quoted at 1.81 CVC-BSI / 1000 CVC-pt days [3]. The rate of inadvertent arterial puncture is quoted at $1 / 1800$ [4].

Use of video in medical education is increasingly common [5] and has been shown to be superior to traditional four-step technique [6].

\section{Objectives}

In response to incidents at Central Manchester Foundation Trust, we produced a series of educational videos to improve our CVC complication rate. Whilst other videos are available, they are of varying quality and we believed it beneficial to demonstrate locally accepted technique, using locally available equipment.

\section{Methods}

Two videos were produced (with patient consent and as part of standard clinical care)

\section{Preparation for inserting CVCs}

2. Inserting internal jugular line with ultrasound

In view of the rarity of complications and our wish to judge effectiveness of the videos we surveyed doctors starting on intensive care on their perceived improvement in all areas of CVC insertion.

${ }^{1}$ Kings College Hospital, UK, London, United Kingdom

Full list of author information is available at the end of the article

\section{Results}

13 doctors were surveyed before and after watching the videos, 8 doctors were senior level (ST3+) and had inserted more than $50 \mathrm{CVCs}$, none reported any perceived improvement. 5 doctor were junior level (ST2 or below) and had limited experience inserting CVCs. $80 \%$ of junior doctors $(n=4)$ reported improved perceived knowledge in inserting CVCs, particularly in required monitoring and post-procedure care.

\section{Conclusion}

The use of medical education videos has been proven and we believe we have made a high quality video which will improve patient safety and the training of junior doctors. The video will be available to view in the eposter.

\section{Grant Acknowledgement}

Funding for recording the videos was received by TeleflexUK. No individual involved in producing the videos received funding from CMFT.

\section{Authors' details}

${ }^{1}$ Kings College Hospital, UK, London, United Kingdom. ${ }^{2}$ North Manchester General Hospital, Manchester, United Kingdom. ${ }^{3}$ Central Manchester Foundation NHS Trust, Manchester, United Kingdom.

Published: 1 October 2015

\section{References}

1. Guidance on the use of USS locating devices for placing CVCS. NICE guidance TA49 2002, Sept.

2. Loveday HP: J Hosp Infect 2014, 86:S1-S70

3. Bion J: "Matching Michigan": a 2-year stepped interventional programme to minimise CVC-blood stream infections in ICUs in England.bmjgs-2012.

4. Guilbert M: Arterial trauma during CVC insertion: Case series, review and proposed algorithm. J Vasc Surg 2008, 48:918-925.

5. McMahon G: Videos in clinical medicine. NEJM 2006, 354:1635.

6. Lee JC: RCT of an instructional DVD for clinical skills teaching. EMA 2007, 19:241-245.

doi:10.1186/2197-425X-3-S1-A869

Cite this article as: Carroll et al.: Use of video technology for teaching in intensive care. Intensive Care Medicine Experimental 2015 3(Suppl 1):A869.

\section{SpringerOpen ${ }^{\circ}$}

C 2015 Carroll et al.; This is an Open Access article distributed under the terms of the Creative Commons Attribution License (http:// creativecommons.org/licenses/by/4.0), which permits unrestricted use, distribution, and reproduction in any medium, provided the original work is properly cited. 